\title{
The Hermitian R-Conjugate Generalized Procrustes Problem
}

\author{
Hai-Xia Chang, ${ }^{1}$ Xue-Feng Duan, ${ }^{2}$ and Qing-Wen Wang ${ }^{3}$ \\ ${ }^{1}$ Department of Applied Mathematics, Shanghai Finance University, Shanghai 201209, China \\ ${ }^{2}$ School of Mathematics and Computational Science, Guilin University of Electronic Technology, Guilin 541004, China \\ ${ }^{3}$ Department of Mathematics, Shanghai University, Shanghai 200444, China
}

Correspondence should be addressed to Hai-Xia Chang; hcychang@163.com

Received 10 May 2013; Accepted 14 August 2013

Academic Editor: Masoud Hajarian

Copyright (C) 2013 Hai-Xia Chang et al. This is an open access article distributed under the Creative Commons Attribution License, which permits unrestricted use, distribution, and reproduction in any medium, provided the original work is properly cited.

\begin{abstract}
We consider the Hermitian $R$-conjugate generalized Procrustes problem to find Hermitian $R$-conjugate matrix $X$ such that $\sum_{k=1}^{p}\left\|A_{k} X-C_{k}\right\|^{2}+\sum_{l=1}^{q}\left\|X B_{l}-D_{l}\right\|^{2}$ is minimum, where $A_{k}, C_{k}, B_{l}$, and $D_{l}(k=1,2, \ldots, p, l=1, \ldots, q)$ are given complex matrices, and $p$ and $q$ are positive integers. The expression of the solution to Hermitian $R$-conjugate generalized Procrustes problem is derived. And the optimal approximation solution in the solution set for Hermitian $R$-conjugate generalized Procrustes problem to a given matrix is also obtained. Furthermore, we establish necessary and sufficient conditions for the existence and the formula for Hermitian $R$-conjugate solution to the linear system of complex matrix equations $A_{1} X=C_{1}, A_{2} X=C_{2}, \ldots, A_{p} X=C_{p}$, $X B_{1}=D_{1}, \ldots, X B_{q}=D_{q}$ ( $p$ and $q$ are positive integers). The representation of the corresponding optimal approximation problem is presented. Finally, an algorithm for solving two problems above is proposed, and the numerical examples show its feasibility.
\end{abstract}

\section{Introduction}

Throughout, let $\mathscr{C}^{m \times n}$ denote the set of all complex $m \times n$ matrices, $\mathscr{R}^{m \times n}$ the set of all real $m \times n$ matrices, and $\mathscr{R}_{r}^{m \times n}$ the set of all matrices in $\mathscr{R}^{m \times n}$ with rank $r$. The symbols $I$, $\bar{A}, A^{T}, A^{*}, A^{\dagger}$, and $\|A\|$, respectively, stand for the identity matrix with the appropriate size, the conjugate, the transpose, the conjugate transpose, the Moore-Penrose inverse, and the Frobenius norm of $A \in \mathscr{C}^{m \times n}$. For $A=\left(a_{i j}\right), B=\left(b_{i j}\right) \in \mathscr{C}^{m \times n}$; $A * B=\left(a_{i j} b_{i j}\right) \in \mathscr{C}^{m \times n}$ represents the Hadamard product of $A$ and $B$.

A linear model of image restoration is a matrix-vector equation is

$$
y=K x+n,
$$

where $y$ represents the observed image, $x$ the original true image, $n$ additive noise, and $K$ a blurring matrix. Image restoration is to minimize blur in an observed image, namely, recover a optimal approximation of $x$ by given $y$ and $K$, and get some statistical information of $n$. The process of the image restoration for the model (1) can be described as

$$
\min _{x}\|x\|^{2} \quad \text { subject to }\|K x-y\|^{2}=\varepsilon,
$$

where $\varepsilon$ is a small positive parameter. It is known that $\widehat{x}=$ $K^{\dagger} y$ is the least squares solution of (1) with minimal norm. However, for $\varepsilon=0$, the solution $\widehat{x}_{\varepsilon}$ to (2), that is, $n=0$ in (1) is not feasible. We know if $\varepsilon \rightarrow 0$, then the solution $\widehat{x}_{\varepsilon}$ converges to $\widehat{x}$. In order to obtain a solution $\widehat{x}_{\varepsilon}$ sufficiently near to $\widehat{x}$, we usually take small $\varepsilon$ such that $0<\varepsilon \ll \delta$, where the error norm $\delta:=\|n\|$ is given.

Now we consider the generalized problem of the process of the image restoration.

Problem 1. Given $\mathcal{S} \subseteq \mathscr{C}^{n \times n}, m_{k}, h_{l}, p, q$ being positive integers, $A_{k}, C_{k} \in \mathscr{C}^{m_{k} \times n}, B_{l}, D_{l} \in \mathscr{C}^{n \times h_{l}}, E \in \mathscr{C}^{n \times n}$, and $k=1, \ldots, p, l=1, \ldots, q$. Let

$$
\mathscr{C}=\left\{X \in \mathcal{S} \mid \sum_{k=1}^{p}\left\|A_{k} X-C_{k}\right\|^{2}+\sum_{l=1}^{q}\left\|X B_{l}-D_{l}\right\|^{2}=\min \right\} .
$$

Find $\widehat{X} \in \mathscr{C}$ such that

$$
\|\widehat{X}-E\|=\min _{X \in \mathscr{C}}\|X-E\| .
$$

The constraint Procrustes problem associated with several kinds of sets $\mathcal{S}$, that is, $p=1$ and $q=0$ in (3) has 
been extensively studied, such as the orthogonal Procrustes problem with $\mathcal{S}$ being the set of orthogonal matrices [1], the symmetric Procrustes problem [2], $(M, N)$-symmetric Procrustes problem [3], Hermitian, Hermitian R-symmetric and Hermitian R-skew-symmetric Procrustes problems [4], the Procrustes problems with $\mathcal{S}$ constrained to the cone of symmetric positive semidefinite and symmetric elementwise matrices [5], and the generalized Procrustes analysis [6]. The optimal approximation problem (4) is initially proposed in the processes of testing or revising given data. A preliminary estimate $E$ of the unknown matrix $X$ in $\mathscr{C}$ can be obtained from experimental observation values and the information of statistical distribution.

We characterize the case $A_{k} X=C_{k}, X B_{l}=D_{l}, k=$ $1, \ldots, p, l=1, \ldots, q$ in Problem 1 and describe it as follows.

Problem 2. Given $\mathcal{S} \subseteq \mathscr{C}^{n \times n}, m_{1}, \ldots, m_{p}, h_{1}, \ldots, h_{q}, p$ and $q$ being positive integers, $A_{1}, C_{1} \in \mathscr{C}^{m_{1} \times n}, A_{2}, C_{2} \in$ $\mathscr{C}^{m_{2} \times n}, \ldots, A_{p}, C_{p} \in \mathscr{C}^{m_{p} \times n}, B_{1}, D_{1} \in \mathscr{C}^{n \times h_{1}}, \ldots, B_{q}, D_{q} \in$ $\mathscr{C}^{n \times h_{q}}, F \in \mathscr{C}^{n \times n}$. Let

$$
\begin{gathered}
\mathscr{L}=\left\{X \in \mathcal{S} \mid A_{1} X=C_{1}, A_{2} X=C_{2}, \ldots, A_{p} X=C_{p},\right. \\
\left.X B_{1}=D_{1}, \ldots, X B_{q}=D_{q}\right\} .
\end{gathered}
$$

When $\mathscr{L}$ is nonempty, find $\widetilde{X} \in \mathscr{L}$ such that

$$
\|\widetilde{X}-F\|=\min _{X \in \mathscr{L}}\|X-F\| .
$$

For important results to solve Problem 2 with different sets $\mathcal{S}$, we refer to [7-14].

Motivated by the work mentioned above, in this paper we mainly discuss the above two problems associated with $S$ being the set of Hermitian $R$-conjugate matrices.

Recall that an $n \times n$ complex matrix $K$ is $R$-conjugate if $\bar{K}=R K R$, where $R \in \mathscr{R}^{n \times n}$ is a nontrivial involution, that is, $R^{2}=I, R \neq \pm I$, which was defined in [15]. A matrix $K \in \mathscr{C}^{n \times n}$ is Hermitian $R$-conjugate if $K^{*}=K$ and $\bar{K}=R K R$, where $R^{T}=R^{-1}=R \neq \pm I$. The Hermitian $R$-conjugate matrix is very useful in scientific computation and digital signal and image processing, its special case, for example, Hermitian Toeplitz matrix, have been studied by several authors, see [14, 16-21]. We denote the set of all $n \times n$ Hermitian $R$-conjugate matrices by $H R_{c} \mathscr{C}^{n \times n}$. Let $R_{c} \mathscr{C}^{n \times n}, S \mathscr{R}^{n \times n}$, and $A S \mathscr{R}^{n \times n}$ denote the set of all $n \times n$ complex $R$-conjugate matrices, real symmetric matrices, real skew-symmetric matrices, respectively.

This paper is organized as follows. In Section 2, we give some preliminary lemmas. In Section 3, we derive the expression of the unique solution to the Problem 1 with $\mathcal{S}=$ $H R_{c} \mathscr{C}^{n \times n}$. In Section 4 , we establish the solvability conditions for existence and an expression of the solution for Problem 2 with $\mathcal{S}=H R_{c} \mathscr{C}^{n \times n}$. In Section 5, we give examples to illustrate the results obtained in this paper.

\section{Preliminaries}

In order to study Problems 1 and 2 with $\mathcal{S}=H R_{c} \mathscr{C}^{n \times n}$, we first give some preliminary lemmas in this section.
For a nontrivial symmetric involutory matrix $R \in \mathscr{R}^{n \times n}$, there exist positive integers $r$ and $s$ such that $r+s=n$ and an $n \times n$ orthogonal matrix $[P, Q]$ such that

$$
R=[P, Q]\left[\begin{array}{cc}
I_{r} & 0 \\
0 & -I_{s}
\end{array}\right]\left[\begin{array}{l}
P^{T} \\
Q^{T}
\end{array}\right],
$$

where $P \in \mathscr{R}^{n \times r}$ and $Q \in \mathscr{R}^{n \times s}$. The columns of $P(Q)$ form an orthogonal basis for the eigenspace of $R$ associated with the eigenvalue $1(-1)$.

Throughout this paper, we always suppose the nontrivial symmetric involutory matrix $R$ is fixed which is given by (7).

Lemma 3 (see Theorem 2.1 in [14]). A matrix $K \in R_{c} \mathscr{C}^{n \times n}$ if and only if there exists $H_{1} \in \mathscr{R}^{n \times n}$ such that $K=U H_{1} U^{*}$, and $K \in H R_{c} \mathscr{C}^{n \times n}$ if and only if there exists $H_{2} \in S \mathscr{R}^{n \times n}$ such that $\mathrm{K}=\mathrm{UH}_{2} \mathrm{U}^{*}$, where

$$
U=[P,-i Q]
$$

with $P$ and $Q$ being the same as (7).

Lemma 4. For any matrix $A \in \mathscr{C}^{n \times n}, A=A_{1} \oplus A_{2} \oplus i A_{3}$, where

$$
\begin{aligned}
& B=\frac{A+R \bar{A} R}{2}, \quad A_{1}=\frac{B+B^{*}}{2}, \\
& A_{2}=\frac{B-B^{*}}{2}, \quad A_{3}=\frac{A-R \bar{A} R}{2 i},
\end{aligned}
$$

and $\oplus$ denotes the direct sum.

Proof. For $B \in \mathscr{C}^{n \times n}$, it is obvious that $B=A_{1} \oplus A_{2}$, where $A_{1}$ are $A_{2}$ are defined in (9). Hence, we just need to prove $A=B \oplus i A_{3}$.

For any matrix $A \in \mathscr{C}^{n \times n}$, it is obvious that $A=B+i A_{3}$, where $B$ and $A_{3}$ are defined in (9).

We prove the uniqueness of $A=B+i A_{3}$. Note that

$$
R B R=\bar{B}, \quad R A_{3} R=\overline{A_{3}},
$$

that is, $B, A_{3} \in R_{c} \mathscr{C}^{n \times n}$. If there exist $D$ and $C_{3}$ satisfying

$$
R D R=\bar{D}, \quad R C_{3} R=\overline{C_{3}}
$$

such that $A=D+i C_{3}$, then

$$
B-D=i\left(C_{3}-A_{3}\right) \text {. }
$$

Multiplying $R$ on the left and right side and then taking the conjugate for (12), it yields

$$
B-D=i\left(A_{3}-C_{3}\right),
$$

implying $B=D$ and $A_{3}=C_{3}$.

So $A=A_{1} \oplus A_{2} \oplus i A_{3}$ holds, where $A_{1}, A_{2}$, and $A_{3}$ are defined in (9).

Lemma 5. Let the symmetric involution $R \in \mathscr{R}^{n \times n}$ be given in (7) and let $U$ be defined as (8). Then 
(i) a matrix $A \in \mathscr{C}^{m \times n}$ satisfies $A R=\bar{A}$ if and only if $A U \in R^{m \times n}$

(ii) a matrix $B \in \mathscr{C}^{n \times l}$ satisfies $R B=\bar{B}$ if and only if $U^{*} B \epsilon$ $R^{n \times l}$.

Proof. (i) It yields from (7) and (8) that

$$
R=[P,-i Q]\left[\begin{array}{cc}
I_{r} & 0 \\
0 & -I_{s}
\end{array}\right]\left[\begin{array}{c}
P^{T} \\
i Q^{T}
\end{array}\right]=U\left[\begin{array}{cc}
I_{r} & 0 \\
0 & -I_{s}
\end{array}\right] U^{*} .
$$

By (14)

$$
\bar{A}=A R=A U\left[\begin{array}{cc}
I_{r} & 0 \\
0 & -I_{s}
\end{array}\right] U^{*},
$$

that is,

$$
A=\overline{A U}\left[\begin{array}{cc}
I_{r} & 0 \\
0 & -I_{s}
\end{array}\right] \bar{U}^{*}=\overline{A U} U^{*},
$$

implying $A U=\overline{A U}$, that is, $A U \in R^{m \times n}$.

Conversely, if $A U \in R^{m \times n}$, according to the proof of the necessity, we can get $\bar{A}=A R$.

The proof of (ii) can be analogously completed according to the proof of (i).

\section{The Solution to Problem 1 with $\mathcal{S}=H R_{c} \mathscr{C}^{n \times n}$}

We, in this section, give the explicit expression of the solution to Problem 1 with $\mathcal{S}=H R_{c} \mathscr{C}^{n \times n}$. In the following, we refer to the $\mathcal{S}=H R_{c} \mathscr{C}^{n \times n}$ in $\mathscr{C}$.

According to Lemma 3, if $X \in H R_{c} \mathscr{C}^{n \times n}$, then

$$
X=U Y U^{*},
$$

where $U$ is defined as (8) and $Y \in S \mathscr{R}^{n \times n}$. Let

$$
\begin{gathered}
A_{k}=A_{k 1}+i A_{k 2} \in \mathscr{C}^{m_{k} \times n}, \\
A_{k 1}=\frac{A_{k}+\overline{A_{k}} R}{2}, \quad A_{k 2}=\frac{A_{k}-\overline{A_{k}} R}{2 i}, \\
B_{l}=B_{l 1}+i B_{l 2} \in \mathscr{C}^{n \times h_{l}}, \\
B_{l 1}=\frac{B_{l}+R \overline{B_{l}}}{2}, \quad B_{l 2}=\frac{B_{l}-R \overline{B_{l}}}{2 i}, \\
C_{k} U=C_{k 1}+i C_{k 2}, \quad C_{k 1}, C_{k 2} \in \mathscr{R}^{m_{k} \times n}, \\
U^{*} D_{l}=D_{l 1}+i D_{l 2}, \quad D_{l 1}, D_{l 2} \in \mathscr{R}^{n \times h_{l}} .
\end{gathered}
$$

By Lemma 5, it is easy to verify $A_{k 1} U, A_{k 2} U \in \mathscr{R}^{m_{k} \times n}$, $U^{*} B_{l 1}, U^{*} B_{l 2} \in \mathscr{R}^{n \times h_{l}}$. We obtain

$$
\begin{aligned}
A_{k} X-C_{k} & =\left(A_{k 1}+i A_{k 2}\right) U Y U^{*}-C_{k} \\
& =\left[\left(A_{k 1} U Y-C_{k 1}\right)+i\left(A_{k 2} U Y-C_{k 2}\right)\right] U^{*}, \\
X B_{l}-D_{l} & =U Y U^{*}\left(B_{l 1}+i B_{l 2}\right)-D_{l} \\
& =U\left[Y U^{*}\left(B_{l 1}-D_{l 1}\right)+i Y U^{*}\left(B_{l 2}-D_{l 2}\right)\right] .
\end{aligned}
$$

It follows from the unitary invariance of Frobenius norm, (21), (22), and $Y \in S \mathscr{R}^{n \times n}$ that

$$
\begin{aligned}
& \sum_{k=1}^{p}\left\|A_{k} X-C_{k}\right\|^{2}+\sum_{l=1}^{q}\left\|X B_{l}-D_{l}\right\|^{2} \\
& =\sum_{k=1}^{p}\left(\left\|A_{k 1} U Y-C_{k 1}\right\|^{2}+\left\|A_{k 2} U Y-C_{k 2}\right\|^{2}\right) \\
& +\sum_{l=1}^{q}\left(\left\|Y U^{*} B_{l 1}-D_{l 1}\right\|^{2}+\left\|Y U^{*} B_{l 2}-D_{l 2}\right\|^{2}\right) \\
& =\sum_{k=1}^{p}\left\|\left[\begin{array}{c}
A_{k 1} U \\
A_{k 2} U
\end{array}\right] Y-\left[\begin{array}{l}
C_{k 1} \\
C_{k 2}
\end{array}\right]\right\|^{2} \\
& +\sum_{l=1}^{q}\left\|Y\left[U^{*} B_{l 1}, U^{*} B_{l 2}\right]-\left[\begin{array}{ll}
D_{l 1}, & D_{l 2}
\end{array}\right]\right\|^{2} \\
& =\sum_{k=1}^{p}\left\|\left[\begin{array}{c}
A_{k 1} U \\
A_{k 2} U
\end{array}\right] Y-\left[\begin{array}{l}
C_{k 1} \\
C_{k 2}
\end{array}\right]\right\|^{2} \\
& +\sum_{l=1}^{q}\left\|\left[\begin{array}{l}
\left(U^{*} B_{l 1}\right)^{T} \\
\left(U^{*} B_{l 2}\right)^{T}
\end{array}\right] Y-\left[\begin{array}{l}
D_{l 1}^{T} \\
D_{l 2}^{T}
\end{array}\right]\right\|^{2} .
\end{aligned}
$$

Suppose

$$
\begin{gathered}
S=\left[\left(A_{11} U\right)^{T},\left(A_{12} U\right)^{T}, \ldots,\left(A_{p 1} U\right)^{T},\left(A_{p 2} U\right)^{T},\right. \\
\left.U^{*} B_{11}, U^{*} B_{12}, \ldots, U^{*} B_{q 1}, U^{*} B_{q 2}\right]^{T}, \\
G=\left[C_{11}^{T}, C_{12}^{T}, \ldots, C_{p 1}^{T}, C_{p 2}^{T}, D_{11}, D_{12}, \ldots, D_{q 1}, D_{q 2}\right]^{T},
\end{gathered}
$$

then

$$
\sum_{k=1}^{p}\left\|A_{k} X-C_{k}\right\|^{2}+\sum_{l=1}^{q}\left\|X B_{l}-D_{l}\right\|^{2}=\|S Y-G\|^{2} .
$$

We first give the following lemma which can be obtained by contrast with Lemma 2.1 in [22].

Lemma 6. Given $S, G \in \mathscr{R}^{\left(m_{1}+\cdots+m_{p}+h_{1}+\cdots+h_{q}\right) \times n}$; therefore, the singular value decomposition (SVD) of $S \in$ $\mathscr{R}_{r_{1}}^{\left(m_{1}+\cdots+m_{p}+h_{1}+\cdots+h_{q}\right) \times n}$ can be described as

$$
S=W\left[\begin{array}{cc}
\Sigma_{r_{1}} & 0 \\
0 & 0
\end{array}\right] V^{T}=W_{1} D_{r_{1}} V_{1}^{T}
$$

where

$$
W=\left[W_{1}, W_{2}\right], \quad V=\left[V_{1}, V_{2}\right],
$$

Let $\quad W_{1} \quad \in \quad \mathscr{R}^{\left(m_{1}+\cdots+m_{p}+h_{1}+\cdots+h_{q}\right) \times r_{1}}, \quad W_{2} \in$ $\mathscr{R}^{\left(m_{1}+\cdots+m_{p}+h_{1}+\cdots+h_{q}\right) \times\left(m_{1}+\cdots+m_{p}+h_{1}+\cdots+h_{q}-r_{1}\right)}, V_{1} \in \mathscr{R}^{n \times r_{1}}$, $V_{2} \in \mathscr{R}^{n \times\left(n-r_{1}\right)}$, and $\Sigma_{r_{1}}=\operatorname{diag}\left(d_{1}, \ldots, d_{r_{1}}\right)$ with $d_{i}>0$, 
$i=1, \ldots, r_{1} ; \Phi=\left(1 /\left(d_{i}^{2}+d_{j}^{2}\right)\right)_{r_{1} \times r_{1}}$. Then $\min _{Y \in S \mathscr{R}^{n \times n}}\|S Y-G\|$ is consistent if and only if

$$
Y=V\left[\begin{array}{cc}
\Phi *\left(\Sigma_{r_{1}} W_{1}^{T} G V_{1}+V_{1}^{T} G^{T} W_{1} \Sigma_{r_{1}}\right) & \Sigma_{r_{1}}^{-1} W_{1}^{T} G V_{2} \\
V_{2}^{T} G^{T} W_{1} \Sigma_{r_{1}}^{-1} & L
\end{array}\right] V^{T},
$$

where $L \in S \mathscr{R}^{\left(n-r_{1}\right) \times\left(n-r_{1}\right)}$ is arbitrary.

Theorem 7. Given $A_{k}, C_{k} \in \mathscr{C}^{m_{k} \times n}, B_{l}, D_{l} \in \mathscr{C}^{n \times h_{l}}$, and positive integers $m_{k}, h_{l}, p$, and $q$, where $k=1, \ldots, p, l=$ $1, \ldots, q$, the notations $A_{k 1}, A_{k 2}, B_{l 1}, B_{l 2}, C_{k 1}, C_{k 2}, D_{l 1}, D_{l 2}$, $S, G$ are defined as (18), (19), (20), (24), and (25), respectively. Let the SVD of $S \in \mathscr{R}_{r_{1}}^{\left(m_{1}+\cdots+m_{p}+h_{1}+\cdots+h_{q}\right) \times n}$ be of the form (27) with (28). Then

$$
\begin{aligned}
& \mathscr{C}=\left\{L \in S \mathscr{R}^{\left(n-r_{1}\right) \times\left(n-r_{1}\right)} \mid U V\right. \\
& \times\left[\begin{array}{cc}
\Phi *\left(\Sigma_{r_{1}} W_{1}^{T} G V_{1}+V_{1}^{T} G^{T} W_{1} \Sigma_{r_{1}}\right) & \Sigma_{r_{1}}^{-1} W_{1}^{T} G V_{2} \\
V_{2}^{T} G^{T} W_{1} \Sigma_{r_{1}}^{-1} & L
\end{array}\right] \\
& \left.\times V^{T} U^{*}\right\} \text {. }
\end{aligned}
$$

Proof. It yields from (26) that

$$
\begin{gathered}
\min _{X \in H R_{c} \mathscr{C}^{\mathscr{g}^{\times n}}} \sum_{k=1}^{p}\left\|A_{k} X-C_{k}\right\|^{2}+\sum_{l=1}^{q}\left\|X B_{l}-D_{l}\right\|^{2} \\
=\min _{Y \in S \mathscr{R}^{n \times n}}\|S Y-G\|^{2} .
\end{gathered}
$$

By Lemma $6, \min _{Y \in S \mathscr{R} \text { nxn }}\|S Y-G\|$ is consistent if and only if $Y$ has the expression of (29). Taking (29) into (17), we obtain the solution set $\mathscr{C}$ is (30).

Theorem 8. Given $E \in \mathscr{C}^{n \times n}$, the equation (4) is consistent if and only if

$$
\begin{aligned}
\widehat{X}= & U V \\
& \times\left[\begin{array}{cc}
\Phi *\left(\Sigma_{r_{1}} W_{1}^{T} G V_{1}+V_{1}^{T} G^{T} W_{1} \Sigma_{r_{1}}\right) & \Sigma_{r_{1}}^{-1} W_{1}^{T} G V_{2} \\
V_{2}^{T} G^{T} W_{1} \Sigma_{r_{1}}^{-1} & \frac{V_{2}^{T} U^{*}\left(E+E^{*}\right) U V_{2}}{2}
\end{array}\right] \\
& \times V^{T} U^{*} .
\end{aligned}
$$

Proof. Obviously, $\mathscr{C}$ is a closed convex set. Hence, there exists the unique element $\widehat{X} \in \mathscr{L}$ such that (4) holds. By applying
Theorem 7 and the unitary invariance of Frobenius norm, for $X \in \mathscr{C}$, we get

$$
\begin{aligned}
& \|X-E\|^{2} \\
& =\| U V\left[\begin{array}{cc}
\Phi *\left(\begin{array}{c}
\left.\sum_{r_{1}} W_{1}^{T} G V_{1}+V_{1}^{T} G^{T} W_{1} \Sigma_{r_{1}}\right) \\
V_{2}^{T} G^{T} W_{1} \Sigma_{r_{1}}^{-1}
\end{array} \Sigma_{r_{1}}^{-1} W_{1}^{T} G V_{2}\right. \\
L
\end{array}\right] \\
& \quad \times V^{T} U^{*}-E \|^{2} \\
& \left.=\| \begin{array}{cc}
\Phi *\left(\Sigma_{r_{1}} W_{1}^{T} G V_{1}+V_{1}^{T} G^{T} W_{1} \Sigma_{r_{1}}\right) & \Sigma_{r_{1}}^{-1} W_{1}^{T} G V_{2} \\
V_{2}^{T} G^{T} W_{1} \Sigma_{r_{1}}^{-1}
\end{array}\right] \\
& \quad-V^{T} U^{*} E U V \|^{2} \\
& =\left\|\Phi *\left(\Sigma_{r_{1}} W_{1}^{T} G V_{1}+V_{1}^{T} G^{T} W_{1} \Sigma_{r_{1}}\right)-V_{1}^{T} U^{*} E U V_{1}\right\|^{2} \\
& +\left\|\Sigma_{r_{1}}^{-1} W_{1}^{T} G V_{2}-V_{1}^{T} U^{*} E U V_{2}\right\|^{2} \\
& +\left\|V_{2}^{T} G^{T} W_{1} \Sigma_{r_{1}}^{-1}-V_{2}^{T} U^{*} E U V_{1}\right\|^{2}+\left\|L-V_{2}^{T} U^{*} E U V_{2}\right\|^{2} .
\end{aligned}
$$

Then $\min _{X \in \mathscr{C}}\|X-E\|$ is equivalent to

$$
\min _{L \in S \mathscr{R}^{\left(n-r_{1}\right) \times\left(n-r_{1}\right)}}\left\|L-V_{2}^{T} U^{*} E U V_{2}\right\| .
$$

(34) holds if and only if

$$
L=\frac{V_{2}^{T} U^{*}\left(E+E^{*}\right) U V_{2}}{2} .
$$

Substituting (35) into $X \in \mathscr{C}$, we get $\widehat{X}$ is (32).

Corollary 9. $\left\|X_{\min }\right\|=\min _{X \in \mathscr{C}}\|X\|$ if and only if

$$
\begin{aligned}
X_{\min }= & U V \\
& \times\left[\begin{array}{cc}
\Phi *\left(\Sigma_{r_{1}} W_{1}^{T} G V_{1}+V_{1}^{T} G^{T} W_{1} \Sigma_{r_{1}}\right) & \Sigma_{r_{1}}^{-1} W_{1}^{T} G V_{2} \\
V_{2}^{T} G^{T} W_{1} \Sigma_{r_{1}}^{-1} & 0
\end{array}\right] \\
& \times V^{T} U^{*} .
\end{aligned}
$$

Remark 10. when $p=1$ and $q=1$ in Theorem 8 , we can derive a result of Theorem 4.1 in [14].

\section{The Solution to Problem 2 with $\mathcal{S}=H R_{c} \mathscr{C}^{n \times n}$}

We refer to $\mathcal{S}=H R_{c} \mathscr{C}^{n \times n}$ in $\mathscr{L}$ in the following text. In this section, we establish necessary and sufficient conditions for the existence and the expression of $\mathscr{L}$. When $\mathscr{L}$ is nonempty, we present the expression of the unique solution to (6).

It follows from (21) that the system $A_{1} X=C_{1}, A_{2} X=$ $C_{2}, \ldots, A_{p} X=C_{p}, X B_{1}=D_{1}, \ldots, X B_{q}=D_{q}$ with unknown $X \in H R_{c} \mathscr{C}^{n \times n}$ is consistent if and only if there exists $Y \in$ $S \mathscr{R}^{n \times n}$ such that $S Y=G$. We first give the following lemma. 
Lemma 11 (see Theorem 1 in [7]). Given $S, G \in$ $R^{\left(m_{1}+\cdots+m_{p}+h_{1}+\cdots+h_{q}\right) \times n}$. Let the SVD of $S \in R_{r_{1}}^{\left(m_{1}+\cdots+m_{p}+h_{1}+\cdots+h_{q}\right) \times n}$ be (27) with (28). Then the matrix equation $S Y=G$ has a symmetric solution if and only if

$$
S S^{\dagger} G=G, \quad G S^{T}=S G^{T}
$$

and the symmetric solution can be expressed as

$$
Y=S^{\dagger} G+\left(I-S^{\dagger} S\right)\left(S^{\dagger} G\right)^{T}+V_{2} Z V_{2}^{T}
$$

where $Z \in S \mathscr{R}^{\left(n-r_{1}\right) \times\left(n-r_{1}\right)}$ is arbitrary.

Theorem 12. Given $A_{1}, C_{1} \in \mathscr{C}^{m_{1} \times n}, A_{2}, C_{2} \in$ $\mathscr{C}^{m_{2} \times n}, \ldots, A_{p}, C_{p} \in \mathscr{C}^{m_{p} \times n}, B_{1}, D_{1} \in \mathscr{C}^{n \times h_{1}}, \ldots, B_{q}, D_{q} \in$ $\mathscr{C}^{n \times h_{q}}$, and positive integers $p$ and $q$. The notations $A_{k 1}$, $A_{k 2}, B_{l 1}, B_{l 2}, C_{k 1}, C_{k 2}, D_{l 1}, D_{l 2}, S, G$ are defined as (18), (19), (20), (24), and (25), respectively. Let the SVD of $S \in \mathscr{R}_{r_{1}}^{\left(m_{1}+\cdots+m_{p}+h_{1}+\cdots+h_{q}\right) \times n}$ be of the form (27) with (28). Then the solution set $\mathscr{L}$ is nonempty if and only if (37) holds, in which case,

$$
\begin{aligned}
\mathscr{L}=\{ & \left\{\in \mathscr{R}^{\left(n-r_{1}\right) \times\left(n-r_{1}\right)} \mid\right. \\
& \left.U\left[S^{\dagger} G+\left(I-S^{\dagger} S\right)\left(S^{\dagger} G\right)^{T}+V_{2} Z V_{2}^{T}\right] U^{*}\right\} .
\end{aligned}
$$

Proof. If the solution set $\mathscr{L}$ is nonempty, then there exists a matrix $X \in H R_{c} \mathscr{C}^{n \times n}$ such that $A_{1} X=C_{1}, A_{2} X=$ $C_{2}, \ldots, A_{p} X=C_{p}, X B_{1}=D_{1}, \ldots, X B_{q}=D_{q}$. We know that $A_{1} X=C_{1}, A_{2} X=C_{2}, \ldots, A_{p} X=C_{p}, X B_{1}=D_{1}, \ldots, X B_{q}=$ $D_{q}$ with $X \in H R_{c} \mathscr{C}^{n \times n}$ is consistent if and only if there exists $Y \in S \mathscr{R}^{n \times n}$ such that $S Y=G$. By Lemma 11, SY $=G$ is solvable for $Y \in S \mathscr{R}^{n \times n}$ if and only if (37) holds, and the expression of the solution is (38). Insert (38) into (17), then we obtain $\mathscr{L}$ is of the form (39).

Conversely, assume (37) holds, according to the proof of the necessity, $A_{1} X=C_{1}, A_{2} X=C_{2}, \ldots, A_{p} X=C_{p}$, $X B_{1}=D_{1}, \ldots, X B_{q}=D_{q}$ is solvable for $X \in H R_{c} \mathscr{C}^{n \times n}$. For $Z \in S \mathscr{R}^{\left(n-r_{1}\right) \times\left(n-r_{1}\right)}$, by Lemma $3, U\left[S^{\dagger} G+\left(I-S^{\dagger} S\right)\left(S^{\dagger} G\right)^{T}+\right.$ $\left.V_{2} Z V_{2}^{T}\right] U^{*} \in H R_{c} \mathscr{C}^{n \times n}$.

Theorem 13. Given $F \in \mathscr{C}^{n \times n}$ and $\mathscr{L}$ is nonempty. Let $F_{1}=$ $(1 / 4)\left[F+R \bar{F} R+(F+R \bar{F} R)^{*}\right]$. Then (6) is consistent for $\widetilde{X} \in \mathscr{L}$ if and only if

$$
\widetilde{X}=U\left[S^{\dagger} G+\left(I-S^{\dagger} S\right)\left(S^{\dagger} G\right)^{T}+V_{2} V_{2}^{T} U F_{1} U^{*} V_{2} V_{2}^{T}\right] U^{*} .
$$

In particular, if $\left\|X_{\mathrm{inf}}\right\|=\min _{X \in \mathscr{L}}\|X\|$, then

$$
X_{\mathrm{inf}}=U\left[S^{\dagger} G+\left(I-S^{\dagger} S\right)\left(S^{\dagger} G\right)^{T}\right] U^{*} .
$$

Proof. By Lemma 4, $F=F_{1} \oplus F_{2} \oplus i F_{3}$, where

$$
\begin{gathered}
F_{1}=\frac{1}{4}\left[F+R \bar{F} R+(F+R \bar{F} R)^{*}\right], \\
F_{2}=\frac{1}{4}\left[F+R \bar{F} R-(F+R \bar{F} R)^{*}\right], \\
F_{3}=\frac{F-R \bar{F} R}{2 i} .
\end{gathered}
$$

When $\mathscr{L}$ is nonempty, for $X \in \mathscr{L}$, we get

$$
\begin{gathered}
\|X-F\|^{2}=\| U\left[S^{\dagger} G+\left(I-S^{\dagger} S\right)\left(S^{\dagger} G\right)^{T}+V_{2} Z V_{2}^{T}\right] U^{*} \\
-\left(F_{1}+F_{2}+i F_{3}\right) \|^{2} \\
=\| S^{\dagger} G+\left(I-S^{\dagger} S\right)\left(S^{\dagger} G\right)^{T}+V_{2} Z V_{2}^{T}-U^{*} F_{1} U \\
-U^{*} F_{2} U-i U^{*} F_{3} U \|^{2} .
\end{gathered}
$$

Since $F_{1} \in H R_{c} \mathscr{C}^{n \times n}, F_{2}, F_{3} \in R_{c} \mathscr{C}^{n \times n}$, by Lemma 3, we obtain $U^{*} F_{1} U \in S \mathscr{R}^{n \times n}, U^{*} F_{2} U, U^{*} F_{3} U \in \mathscr{R}^{n \times n}$. Note that $U^{*} F_{2} U \in$ $A S \mathscr{R}^{n \times n}$, then

$$
\begin{aligned}
\|X-F\|^{2}= & \left\|U F_{1} U^{*}-S^{\dagger} G-\left(I-S^{\dagger} S\right)\left(S^{\dagger} G\right)^{T}-V_{2} Z V_{2}^{T}\right\|^{2} \\
& +\left\|U F_{2} U^{*}\right\|^{2}+\left\|U F_{3} U^{*}\right\|^{2} .
\end{aligned}
$$

Hence, $\min _{X \in \mathscr{L}}\|X-F\|$ is equivalent to

$$
\min _{Z \in S \mathscr{R}^{\left(n-r_{1}\right) \times\left(n-r_{1}\right)}}\left\|U F_{1} U^{*}-S^{\dagger} G-\left(I-S^{\dagger} S\right)\left(S^{\dagger} G\right)^{T}-V_{2} Z V_{2}^{T}\right\| .
$$

For the orthogonal matrix $V$, we get

$$
\begin{aligned}
& \left\|U F_{1} U^{*}-S^{\dagger} G-\left(I-S^{\dagger} S\right)\left(S^{\dagger} G\right)^{T}-V_{2} Z V_{2}^{T}\right\| \\
& =\left\|V^{T}\left[U F_{1} U^{*}-S^{\dagger} G-\left(I-S^{\dagger} S\right)\left(S^{\dagger} G\right)^{T}-V_{2} Z V_{2}^{T}\right] V\right\| \\
& =\left\|V_{1}^{T}\left[U F_{1} U^{*}-S^{\dagger} G-\left(I-S^{\dagger} S\right)\left(S^{\dagger} G\right)^{T}\right] V_{1}\right\| \\
& \quad+\left\|V_{1}^{T}\left[U F_{1} U^{*}-S^{\dagger} G-\left(I-S^{\dagger} S\right)\left(S^{\dagger} G\right)^{T}\right] V_{2}\right\| \\
& +\left\|V_{2}^{T}\left[U F_{1} U^{*}-S^{\dagger} G-\left(I-S^{\dagger} S\right)\left(S^{\dagger} G\right)^{T}\right] V_{1}\right\| \\
& \quad+\left\|V_{2}^{T}\left[U F_{1} U^{*}-S^{\dagger} G-\left(I-S^{\dagger} S\right)\left(S^{\dagger} G\right)^{T}\right] V_{2}-Z\right\| .
\end{aligned}
$$




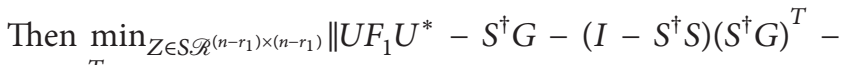
$V_{2} Z V_{2}^{T} \|$ is solvable if and only if

$$
Z=V_{2}^{T}\left[U F_{1} U^{*}-S^{\dagger} G-\left(I-S^{\dagger} S\right)\left(S^{\dagger} G\right)^{T}\right] V_{2} .
$$

It follows from (27) that

$$
S^{\dagger}=V_{1} D_{r_{1}}^{-1} W_{1}^{T}
$$

By (48) and (47) we get

$$
Z=V_{2}^{T} U F_{1} U^{*} V_{2}
$$

Hence, from (39) and (49), we obtain $\widetilde{X}$ which can be expressed as (40).

Remark 14. When $p=1$ and $q=1$ in Theorem 13, we can get a conclusion of Theorem 3.1 in [14].

\section{Numerical Examples}

We, in this section, propose an algorithm for finding the solution of Problems 1 and 2 with $\mathcal{S}=H R_{c} \mathscr{C}^{n \times n}$ and give illustrative numerical examples.
Let the symmetric involutory matrix $R=\left[\begin{array}{ccc}0 & 0 & V_{2} \\ 0 & I_{2} & 0 \\ V_{2} & 0 & 0\end{array}\right] \epsilon$ $\mathscr{R}^{6 \times 6}$, where $V_{2}=\left[\begin{array}{ll}0 & 1 \\ 1 & 0\end{array}\right]$. Applying the spectral decomposition of $R$, we obtain the orthogonal matrix [ $P, Q]$ satisfying $(7)$ and then by (8), we get

$$
U=\frac{1}{\sqrt{2}}\left[\begin{array}{cccccc}
0 & 0 & 1 & 0 & 0 & -i \\
0 & 0 & 0 & 1 & i & 0 \\
\sqrt{2} & 0 & 0 & 0 & 0 & 0 \\
0 & \sqrt{2} & 0 & 0 & 0 & 0 \\
0 & 0 & 0 & 1 & -i & 0 \\
0 & 0 & 1 & 0 & 0 & i
\end{array}\right]
$$

Algorithm 15. (1) Input $A_{1}, A_{2}, \ldots, A_{p}, C_{1}, C_{2}, \ldots, C_{p}$, $B_{1}, \ldots, B_{q}, D_{1}, \ldots, D_{q}$;

(2) compute $A_{k 1}, A_{k 2}, B_{l 1}, B_{l 2}, C_{k 1}, C_{k 2}, D_{l 1}, D_{l 2}, S, G$ by (18), (19), (20), (24), and (25);

(3) make the SVD of $S$ with the form of (27) and compute $W_{1}, W_{2}, V_{1}, V_{2}$ by (28);

(4) if (37) holds, continue, or go to step 6;

(5) input $E \in \mathscr{C}^{n \times n}$, compute the solution to Problem 2 with $\mathcal{S}=H R_{c} \mathscr{C}^{n \times n}$ by (40);

(6) input $F \in \mathscr{C}^{n \times n}$, compute the solution of Problem 1 with $\mathcal{S}=H R_{c} \mathscr{C}^{n \times n}$ by (32).

Example 1. We consider the case of $p=1$ and $q=1$. Suppose $A, C \in \mathscr{C}^{5 \times 6}, B, D \in \mathscr{C}^{6 \times 4}$, and

$$
\begin{aligned}
& A=\left[\begin{array}{cccccc}
-2.2163+1.2010 i & 2.2524-1.1328 i & 0 & -0.0955+1.3420 i & 4.3215-2.3145 i & 1.2120+1.9804 i \\
-1.1625 & 3.2510 i & 0 & 2.1800-0.3125 i & -0.2235+1.4140 i & 5.7071 \\
1.0500-2.0120 i & 2.4020+1.2500 i & 0 & 3.2106 i & -1.6080 & 2.2560 \\
0 & 0 & 0 & 0 & 0 & 0 \\
3.2000 & -4.1955+1.2000 i & 0 & 1.1025-0.2400 i & -0.8902-0.2408 i & 1.2010+0.7071 i
\end{array}\right], \\
& C=\left[\begin{array}{cccccc}
4.1365+2.6614 i & 1.5828-0.2259 i & 2.2091+1.5678 i & 2.4154 i & -1.8939+0.4692 i & 2.5155-0.7604 i \\
1.0118-0.2844 i & -3.4235+1.5798 i & 1.3798-0.7942 i & 1.3050+2.9900 i & -2.7833+0.0592 i & 1.8744-0.7889 i \\
0 & 0 & 0 & 0 & 0 & 0 \\
2.1991-1.0648 i & -1.5298 i & 5.6808+1.6029 i & -4.0150+2.1200 i & 2.4387-1.9600 i & 3.4200-2.6288 i \\
2.9883 & 2.4329-0.6405 i & 1.4611-1.0503 i & 3.7680-1.4980 i & -2.0266-0.9334 i & 1.5756 i
\end{array}\right], \\
& B=\left[\begin{array}{cccc}
0.5200+0.7200 i & -0.2000 & 0.4500+0.3600 i & 0 \\
-0.3394-0.5091 i & 0.1414-0.3536 i & -0.3200-0.6500 i & 0 \\
0.5940+0.2970 i & 0.0707+0.0354 i & 0.0700 & 0 \\
0.0200 i & 0.2500 & 0.3500+0.8400 i & 0 \\
-0.3960-0.5091 i & 0.1414+0.3536 i & -0.3200+0.2400 i & 0 \\
0.5940+0.2970 i & 0.2121+0.0354 i & 0.0707 & 0
\end{array}\right] \\
& D=\left[\begin{array}{cccc}
-0.3140+0.1069 i & 0.4954-0.0615 i & 0.7469+0.4876 i & -0.0662+0.0817 i \\
0.4255-0.5754 i & 0.0348+0.5182 i & -0.2522+0.5751 i & -0.0105-0.0928 i \\
0.0883+0.0649 i & 0.2201+0.0182 i & 0.3133+0.3271 i & 0.0076-0.0138 i \\
0.8675+0.7972 i & -0.3105-0.0837 i & -0.1461+0.2541 i & 0.2620+0.0983 i \\
-0.4591+0.6268 i & -0.0405-0.6447 i & 0.1517-0.6198 i & 0.0256+0.2057 i \\
0.1439-0.2759 i & 0.4722+0.1091 i & 0.4952+0.3680 i & -0.0414-0.1178 i
\end{array}\right] .
\end{aligned}
$$




$$
E=\left[\begin{array}{cccccc}
0.4424+0.6274 i & -0.1765-0.0108 i & 0.4006+0.3308 i & 0.3069 & -0.1505+0.1262 i & 1.200+0.0045 i \\
0.6374-0.0108 i & 0.2246-0.2738 i & 0.1509-0.4327 i & -0.0434-0.0915 i & 0.1385-0.1800 i & 0 \\
-0.4950-0.4172 i & 0.1414-0.0354 i & -0.3818-0.1750 i & -0.2475+0.0619 i & -0.1630-0.0540 i & 0.2032 i \\
-0.5129-0.4025 i & 0.1956-0.0157 i & 0.0868+0.5565 i & -0.1227 & -0.2382-0.0911 i & 0 \\
0.1878+0.3187 i & 0.1789+0.3334 i & 0.0271+0.7668 i & -0.0434+0.0915 i & -0.1024 & -0.0002+0.0150 i \\
-0.2404-0.6010 i & 0.1414+0.0354 i & -0.2546-0.3341 i & -0.2475-0.0619 i & -0.0002-0.0150 i & 0.0256
\end{array}\right],
$$

applying Algorithm 15, we get the following results:

$$
\widehat{X}=\left[\begin{array}{cccccc}
-0.1949+0.0000 i & -0.0395+0.3097 i & 0.1067+0.0353 i & 0.6067-0.2888 i & 0.0380-0.6272 i & -0.1218+0.1242 i \\
-0.0395-0.3097 i & -0.7354+0.0000 i & 0.0606-0.1460 i & -0.1095+0.4086 i & 0.6954-0.0593 i & 0.0380-0.6272 i \\
0.1067-0.0353 i & 0.0606+0.1460 i & 0.0009 & 0.3957 & 0.0606-0.1460 i & 0.1067+0.0353 i \\
0.6067+0.2888 i & -0.1095-0.4086 i & 0.3957 & -0.1562 & -0.1095+0.4086 i & 0.6067-0.2888 i \\
0.0380+0.6272 i & 0.6954+0.0593 i & 0.0606+0.1460 i & -0.1095-0.4086 i & -0.7354-0.0000 i & -0.0395+0.3097 i \\
-0.1218-0.1242 i & 0.0380+0.6272 i & 0.1067-0.0353 i & 0.6067+0.2888 i & -0.0395-0.3097 i & -0.1949-0.0000 i
\end{array}\right],
$$

$$
\begin{aligned}
& \|A \widehat{X}-C\|^{2}+\|\widehat{X} B-D\|^{2}=\min _{X \in H R_{c} \mathbb{C}^{6 \times 6}}\|A X-C\|^{2}+\|X B-D\|^{2}=160.0357 \\
& \left\|\widehat{X}-\widehat{X}^{*}\right\|=4.1192 \times 10^{-16}, \quad\|\bar{X}-R X R\|=0, \quad\|\widehat{X}-E\|=3.7397 .
\end{aligned}
$$

The following example is about Problem 2 with $\mathcal{S}=$ $H R_{c} \mathscr{C}^{6 \times 6}, p=1$ and $q=0$. We list results of comparison of the solutions computed by Algorithm 15 and MATLAB procedure $X=A \backslash C$.
Example 2. Let $W_{a} \in \mathscr{C}^{8 \times 8}, V_{a} \in \mathscr{C}^{6 \times 6}$ be unitary matrices,

$$
\begin{aligned}
& W_{a}=\left[\begin{array}{ccccc}
-0.1590+0.2812 i & -0.1803-0.1822 i & 0.4873+0.0557 i & 0.1272+0.2856 i & : \\
0.1159-0.1077 i & 0.4983-0.2783 i & 0.4127-0.2359 i & 0.3195+0.2342 i & : \\
0.2197-0.1364 i & 0.1146-0.1466 i & 0.2900+0.0440 i & -0.5309-0.0423 i & : \\
0.0016-0.0047 i & 0.0108-0.0047 i & -0.0257+0.0018 i & -0.0181+0.0126 i & : \\
0.2576-0.0271 i & 0.2935+0.2509 i & -0.3014+0.0304 i & 0.3562+0.0778 i & : \\
0.4283+0.1012 i & 0.0858-0.1579 i & -0.1758-0.3913 i & -0.5198+0.0316 i & : \\
0.3884-0.2103 i & 0.2167+0.2285 i & 0.1236-0.0447 i & 0.1348-0.0089 i & : \\
0.5625+0.1774 i & -0.2720-0.4682 i & -0.0047+0.3918 i & 0.1951+0.0273 i & :
\end{array}\right. \\
& \left.\begin{array}{cccc}
-0.5270-0.1794 i & 0.1734-0.2471 i & 0.2466+0.1275 i & 0.0437+0.1077 i \\
0.0562-0.0163 i & -0.2264+0.4255 i & -0.1461+0.0547 i & -0.0662-0.0426 i \\
-0.1748+0.4297 i & 0.4788+0.1119 i & -0.1543-0.2114 i & 0.0422-0.0388 i \\
-0.0011-0.0201 i & 0.0249+0.0695 i & 0.0506+0.2945 i & 0.7638-0.5658 i \\
-0.3766-0.0582 i & 0.3605-0.2131 i & -0.4447+0.1694 i & -0.0868-0.0677 i \\
-0.1860-0.3130 i & -0.2654-0.1257 i & 0.0594+0.2924 i & -0.0976+0.0376 i \\
0.3112-0.2036 i & 0.3628-0.1215 i & 0.5860-0.0319 i & 0.1016+0.1755 i \\
0.2087-0.1071 i & -0.0638-0.1313 i & -0.1736-0.2252 i & 0.0555-0.0788 i
\end{array}\right] \\
& V_{a}=\left[\begin{array}{cccccc}
0.4621 & 0.1052 & -0.6218 & -0.1836 & -0.5897 & 0.0852 \\
-0.2474-0.0088 i & -0.1896-0.5726 i & 0.1951-0.1974 i & -0.5870-0.1096 i & -0.2811+0.1327 i & -0.2113-0.0036 i \\
0.4418-0.0309 i & -0.2001-0.2357 i & 0.0004+0.2178 i & 0.0696+0.1631 i & 0.1926-0.3664 i & -0.6629-0.1362 i \\
-0.1299-0.4383 i & 0.1856+0.1319 i & 0.1214-0.4193 i & 0.0858+0.5057 i & -0.2366-0.1016 i & -0.0917-0.4592 i \\
-0.2390+0.2258 i & -0.1938-0.3803 i & 0.0533+0.3571 i & 0.3007+0.3102 i & -0.3167-0.3755 i & 0.3810-0.0794 i \\
0.4263+0.1739 i & 0.3926-0.3760 i & 0.3391-0.2301 i & 0.2360+0.2699 i & -0.0153+0.2763 i & 0.0807+0.3359 i
\end{array}\right],
\end{aligned}
$$


TABLE 1: Comparison of results by Algorithm 15 and the MATLAB procedure $A \backslash C$.

\begin{tabular}{lcccccccc}
\hline$t$ & $e_{1}$ & $e_{2}$ & $e_{3}$ & $e_{4}$ & $t e_{1}$ & $t e_{2}$ & $t e_{3}$ \\
\hline-13 & $2 \cdot 10^{-15}$ & $1 \cdot 10^{-15}$ & 0 & $2 \cdot 10^{-15}$ & $3 \cdot 10^{-15}$ & $3 \cdot 10^{-2}$ & $5 \cdot 10^{-2}$ \\
-12 & $2 \cdot 10^{-15}$ & $1 \cdot 10^{-15}$ & 0 & $1 \cdot 10^{-15}$ & $3 \cdot 10^{-15}$ & $3 \cdot 10^{-3}$ & $5 \cdot 10^{-3}$ & $4 \cdot 10^{-3}$ \\
-11 & $2 \cdot 10^{-15}$ & $2 \cdot 10^{-15}$ & 0 & $1 \cdot 10^{-15}$ & $3 \cdot 10^{-15}$ & $3 \cdot 10^{-4}$ & $4 \cdot 10^{-4}$ \\
-10 & $2 \cdot 10^{-15}$ & $1 \cdot 10^{-15}$ & 0 & $2 \cdot 10^{-15}$ & $3 \cdot 10^{-15}$ & $3 \cdot 10^{-5}$ & $3 \cdot 10^{-4}$ \\
-9 & $3 \cdot 10^{-15}$ & $2 \cdot 10^{-15}$ & 0 & $2 \cdot 10^{-15}$ & $2 \cdot 10^{-15}$ & $3 \cdot 10^{-6}$ & $5 \cdot 10^{-6}$ & $4 \cdot 10^{-5}$ \\
-8 & $2 \cdot 10^{-15}$ & $1 \cdot 10^{-15}$ & 0 & $2 \cdot 10^{-15}$ & $2 \cdot 10^{-15}$ & $3 \cdot 10^{-7}$ & $4 \cdot 10^{-7}$ \\
-7 & $3 \cdot 10^{-15}$ & $2 \cdot 10^{-15}$ & 0 & $1 \cdot 10^{-15}$ & $2 \cdot 10^{-15}$ & $4 \cdot 10^{-8}$ & $5 \cdot 10^{-8}$ \\
-6 & $2 \cdot 10^{-15}$ & $2 \cdot 10^{-15}$ & 0 & $2 \cdot 10^{-15}$ & $2 \cdot 10^{-15}$ & $2 \cdot 10^{-9}$ & $4 \cdot 10^{-9}$ \\
-5 & $2 \cdot 10^{-15}$ & $1 \cdot 10^{-15}$ & 0 & $2 \cdot 10^{-15}$ & $2 \cdot 10^{-15}$ & $2 \cdot 10^{-10}$ & $3 \cdot 10^{-10}$ \\
-4 & $2 \cdot 10^{-15}$ & $1 \cdot 10^{-15}$ & 0 & $1 \cdot 10^{-15}$ & $2 \cdot 10^{-15}$ & $3 \cdot 10^{-11}$ & $3 \cdot 10^{-9}$ \\
-3 & $2 \cdot 10^{-15}$ & $1 \cdot 10^{-15}$ & 0 & $2 \cdot 10^{-15}$ & $3 \cdot 10^{-15}$ & $3 \cdot 10^{-12}$ & $5 \cdot 10^{-12}$ \\
-2 & $2 \cdot 10^{-15}$ & $1 \cdot 10^{-15}$ & 0 & $2 \cdot 10^{-15}$ & $2 \cdot 10^{-15}$ & $2 \cdot 10^{-13}$ & $3 \cdot 10^{-13}$ \\
-1 & $2 \cdot 10^{-15}$ & $1 \cdot 10^{-15}$ & 0 & $2 \cdot 10^{-15}$ & $3 \cdot 10^{-15}$ & $3 \cdot 10^{-14}$ & $5 \cdot 10^{-12}$ \\
0 & $2 \cdot 10^{-15}$ & $2 \cdot 10^{-15}$ & 0 & $2 \cdot 10^{-15}$ & $4 \cdot 10^{-15}$ & $4 \cdot 10^{-15}$ & $6 \cdot 10^{-15}$ & $5 \cdot 10^{-14}$ \\
1 & $9 \cdot 10^{-15}$ & $2 \cdot 10^{-15}$ & 0 & $2 \cdot 10^{-15}$ & $1 \cdot 10^{-14}$ & $5 \cdot 10^{-15}$ & $6 \cdot 10^{-15}$ \\
\hline
\end{tabular}

$$
X=\left[\begin{array}{cccccc}
0.2543 & -0.3625-1.1647 i & -0.1609-0.2793 i & 0.8839-0.4914 i & 0.5675-0.0043 i & -0.4143-0.4378 i \\
-0.3625+1.1647 i & -1.3886 & 0.0672-0.6470 i & -0.2316+0.2397 i & 0.0524-0.4418 i & 0.5675-0.0043 i \\
-0.1609+0.2793 i & 0.0672+0.6470 i & -0.4312 & -0.2500 & 0.0672-0.6470 i & -0.1609-0.2793 i \\
0.8839+0.4914 i & -0.2316-0.2397 i & -0.2500 & -0.0380 & -0.2316+0.2397 i & 0.8839-0.4914 i \\
0.5675+0.0043 i & 0.0524+0.4418 i & 0.0672+0.6470 i & -0.2316-0.2397 i & -1.3886 & -0.3625-1.1647 i \\
-0.4143+0.4378 i & 0.5675+0.0043 i & -0.1609+0.2793 i & 0.8839+0.4914 i & -0.3625+1.1647 i & 0.2543
\end{array}\right]
$$

Then compute $C(t)=A(t) X$ for $t=$ $10,1,10^{-1}, 10^{-2}, \ldots, 10^{-13}$. Obviously, Problem 2 with $\mathcal{S}=H R_{c} \mathscr{C}^{n \times n}$ is consistent for each value of $t$. For matrices $A, C$ obtained above, we first use Algorithm 15 to obtain the Hermitian $R$-conjugate solutions approximate to $X$, then compute the solutions of $A X=C$ by MATLAB procedure $X=A \backslash C$. Let $\widetilde{X}_{i} s$ denote the solutions computed by Algorithm 15 and $X_{i} s$ the solutions by MATLAB procedure $X=A \backslash C$. Let

$$
\begin{array}{cc}
e 1=\left\|A \widetilde{X}_{i}-C\right\|, & e 2=\left\|\widetilde{X}_{i}-X\right\|, \\
e 3=\left\|\widetilde{X}_{i}-R \widetilde{X}_{i} R\right\|, & e 4=\left\|\widetilde{X}_{i}^{T}-\widetilde{X}_{i}\right\|, \\
t e 1=\left\|A X_{i}-C\right\|, & t e 2=\left\|X_{i}-X\right\|, \\
t e 3=\left\|\overline{X_{i}}-R X_{i} R\right\|, & t e 4=\left\|X_{i}^{T}-X_{i}\right\| .
\end{array}
$$

Analysis of Results. As a general observation from Table 1, we find that the performance of Algorithm 15 to solve Problem 2 is very good and that of the MATLAB procedure $X=$ $A \backslash C$ is quite sensitive to the conditioning of matrix $A$. For $t=10,1, \ldots, 10^{-4}$, both methods behave well. In this case we should choose MATLAB procedure $X=A \backslash C$ and $D(t)=\left[\begin{array}{c}D \\ \operatorname{zeros}(2,6)\end{array}\right]$, where $D=\operatorname{diag}(3,2,1, t / 1, t / 2, t / 3)$ and zeros $(2,6)$ is a $2 \times 6$ zeros matrix. Let $A(t)=W_{a} D(t) V_{a}^{*}$ and $X \in H R_{c} \mathscr{C}^{6 \times 6}$,

to solve Problem 2 with $\mathcal{S}=H R_{c} \mathscr{C}^{n \times n}$ for it is simple. However, as some singular values of $A$ are close to zero, the solutions $X_{i}$ computed by MATLAB procedure $X=A \backslash C$ do not satisfy $A X=C$ and gradually lose the property of Hermitian $R$-conjugate matrix, while Algorithm 15 does it well. Hence, when $A$ has small singular values close to zero, the Algorithm 15 predominates over MATLAB procedure $X=A \backslash C$.

\section{Conclusion}

In this paper, we converted the Hermitian $R$-conjugate generalized Procrustes problem to real symmetric Procrustes problem trickly and obtained its solution set. We also investigated the Hermitian $R$-conjugate solution to the linear system of complex matrix equations $A_{1} X=C_{1}, A_{2} X=C_{2}, \ldots, A_{p} X=$ $C_{p}, X B_{1}=D_{1}, \ldots, X B_{q}=D_{q}$ and established solvable conditions and the formula for the Hermitian $R$-conjugate solution. Moreover, we showed the optimal approximation solution to a given matrix in the above two corresponding solution set is unique, respectively. As applications, a numerical algorithm has been given and the examples have illustrated the feasibility of the algorithm. Additionally, we can further consider the least square Hermitian $R$-conjugate solution of 
the system $A_{k} X A_{k}^{*}=C_{k}, k=1,2, \ldots, n$ ( $n$ is positive integer) and the corresponding optimal approximation problem.

\section{Acknowledgments}

The authors are very much indebted to the anonymous referees for their constructive and valuable comments and suggestions which greatly improved the original paper. This research was supported by the Innovation Foundation of Shanghai Municipal Education Commission (13ZZ080), the National Natural Science Foundation of China (11391240185), the Shanghai University Scientific Selection and Cultivation for Outstanding Young Teachers in special fund (sjr10009), and the Natural Science Foundation of Guangxi Province (no. 2012GXNSFBA053006).

\section{References}

[1] B. F. Green, "The orthogonal approximation of an oblique structure in factor analysis," Psychometrika, vol. 17, pp. 429-440, 1952.

[2] N. J. Higham, “The symmetric Procrustes problem,” BIT Numerical Mathematics, vol. 28, no. 1, pp. 133-143, 1988.

[3] J. Peng, X.-Y. Hu, and L. Zhang, "The $(M, N)$-symmetric Procrustes problem," Applied Mathematics and Computation, vol. 198, no. 1, pp. 24-34, 2008.

[4] W. F. Trench, "Hermitian, Hermitian $R$-symmetric, and Hermitian $R$-skew symmetric Procrustes problems," Linear Algebra and Its Applications, vol. 387, pp. 83-98, 2004.

[5] L.-E. Andersson and T. Elfving, "A constrained Procrustes problem," SIAM Journal on Matrix Analysis and Applications, vol. 18, no. 1, pp. 124-139, 1997.

[6] J. C. Gower, "Generalized Procrustes analysis," Psychometrika, vol. 40, pp. 33-51, 1975.

[7] H. Dai, "On the symmetric solutions of linear matrix equations," Linear Algebra and Its Applications, vol. 131, pp. 1-7, 1990.

[8] Z.-Y. Peng and X.-Y. Hu, "The reflexive and anti-reflexive solutions of the matrix equation $A X=B$," Linear Algebra and Its Applications, vol. 375, pp. 147-155, 2003.

[9] R. A. Horn, V. V. Sergeichuk, and N. Shaked-Monderer, "Solution of linear matrix equations in a *congruence class," Electronic Journal of Linear Algebra, vol. 13, pp. 153-156, 2005.

[10] G. K. Hua, X. Hu, and L. Zhang, "A new iterative method for the matrix equation $A X=B$," Applied Mathematics and Computation, vol. 15, pp. 1434-1441, 2007.

[11] A. D. Porter, "Solvability of the matrix equation $A X=B$," Linear Algebra and Its Applications, vol. 13, no. 3, pp. 177-184, 1976.

[12] Q.-W. Wang and J. Yu, "On the generalized bi (skew-) symmetric solutions of a linear matrix equation and its procrust problems," Applied Mathematics and Computation, vol. 219, no. 19, pp. 9872-9884, 2013.

[13] Y. S. Hanna, "On the solutions of tridiagonal linear systems," Applied Mathematics and Computation, vol. 189, no. 2, pp. 20112016, 2007.

[14] C.-Z. Dong, Q.-W. Wang, and Y.-P. Zhang, "On the Hermitian $R$-conjugate solution of a system of matrix equations," Journal of Applied Mathematics, vol. 2012, Article ID 398085, 14 pages, 2012.

[15] W. F. Trench, "Characterization and properties of matrices with generalized symmetry or skew symmetry," Linear Algebra and Its Applications, vol. 377, pp. 207-218, 2004.
[16] T. Huckle, S. Serra-Capizzano, and C. Tablino-Possio, "Preconditioning strategies for Hermitian indefinite Toeplitz linear systems," SIAM Journal on Scientific Computing, vol. 25, no. 5, pp. 1633-1654, 2004.

[17] R. H. Chan, A. M. Yip, and M. K. Ng, "The best circulant preconditioners for Hermitian Toeplitz systems," SIAM Journal on Numerical Analysis, vol. 38, no. 3, pp. 876-896, 2000.

[18] A. Lee, "Centro-Hermitian and skew-centro-Hermitian matrices," Linear Algebra and Its Applications, vol. 29, pp. 205-210, 1980.

[19] A. V. Oppenheim, Applications of Digital Signal Processing, Prentice-Hall, Englewood Cliffs, Calif, USA, 1978.

[20] M. K. Ng, R. J. Plemmons, and F. Pimentel, "A new approach to constrained total least squares image restoration," Linear Algebra and Its Applications, vol. 316, no. 1-3, pp. 237-258, 2000.

[21] R. Kouassi, P. Gouton, and M. Paindavoine, "Approximation of the Karhunen-Loève tranformation and its application to colour images," Signal Processing: Image Commu, vol. 16, pp. 541-551, 2001.

[22] Y.-B. Deng, X.-Y. Hu, and L. Zhang, "Least squares solution of $B X A^{T}=T$ over symmetric, skew-symmetric, and positive semidefinite X," SIAM Journal on Matrix Analysis and Applications, vol. 25, no. 2, pp. 486-494, 2003. 


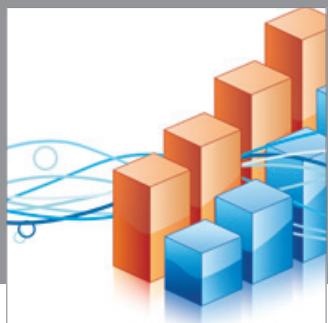

Advances in

Operations Research

mansans

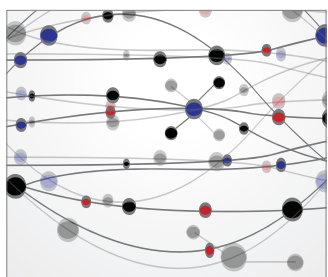

The Scientific World Journal
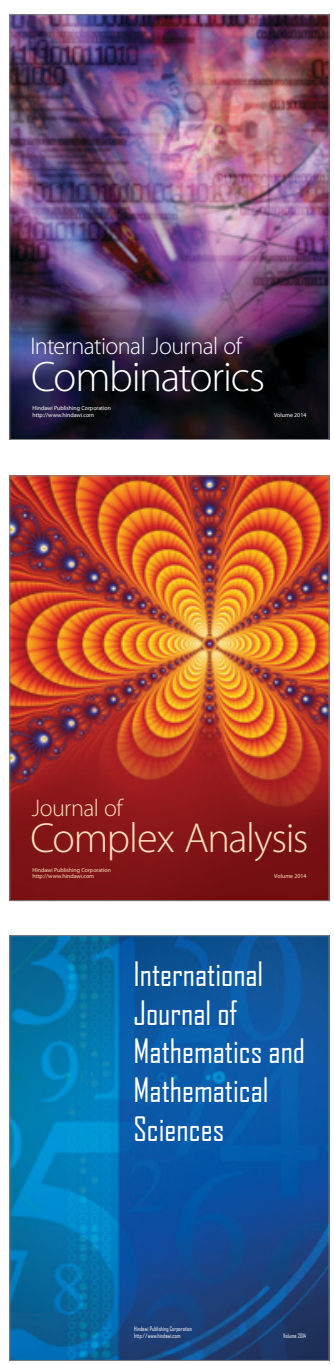
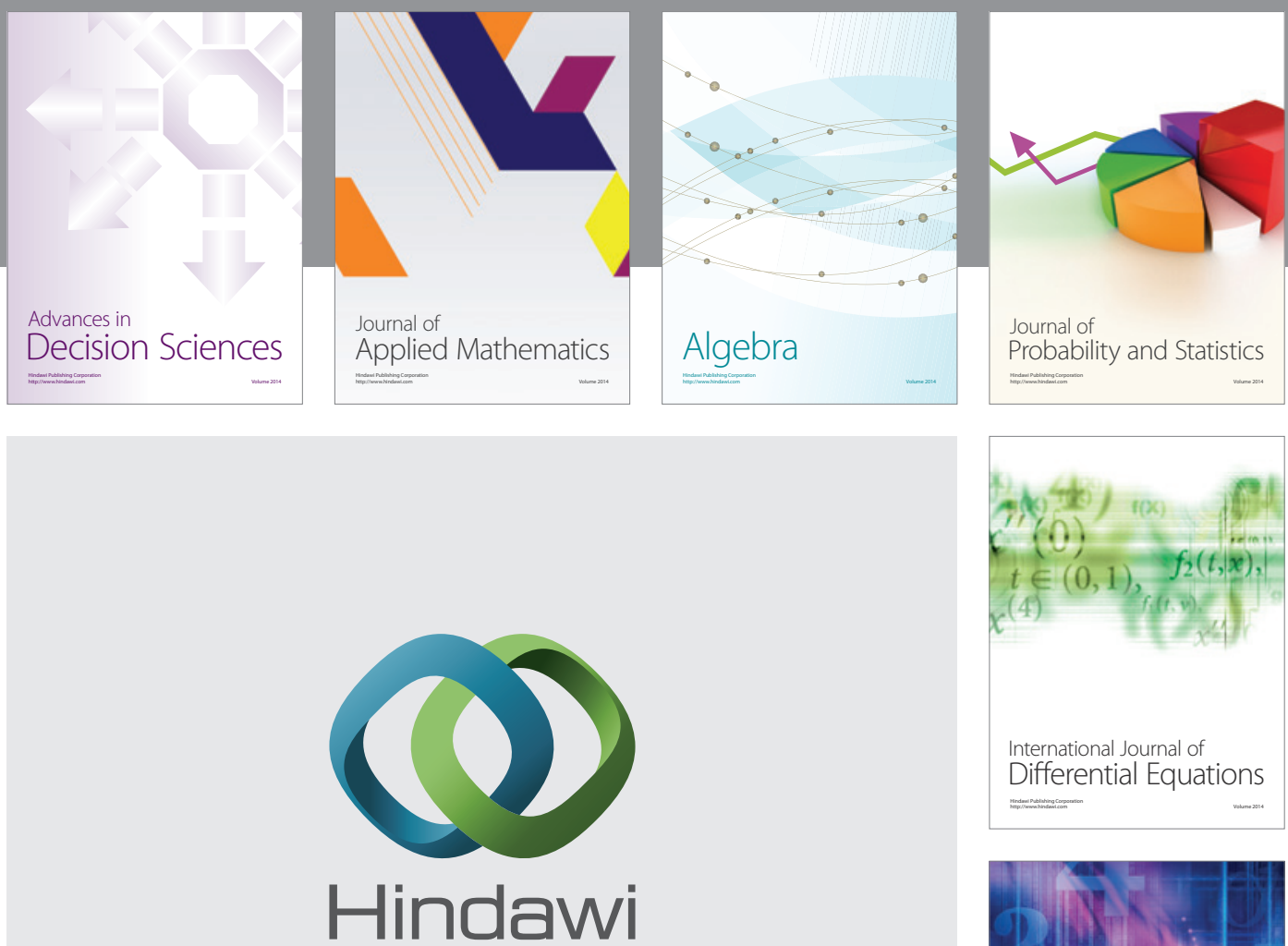

Submit your manuscripts at http://www.hindawi.com
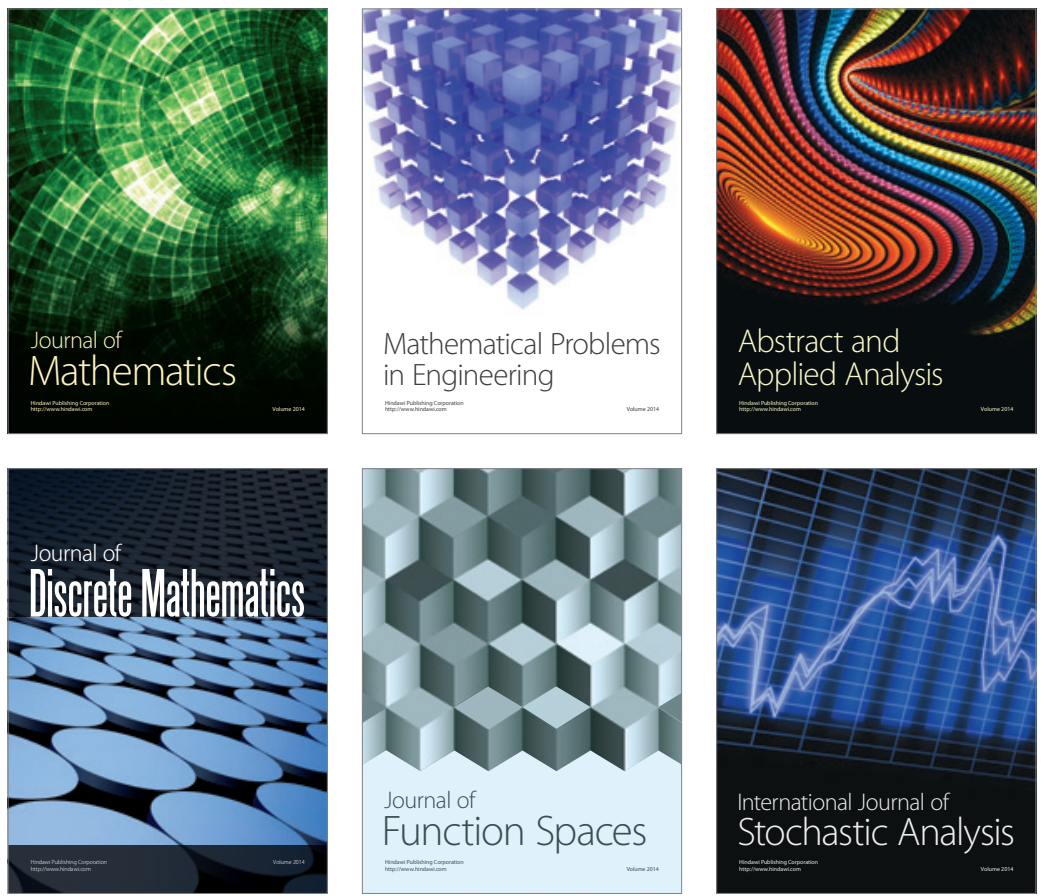

Journal of

Function Spaces

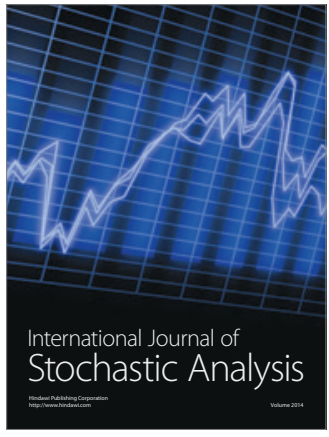

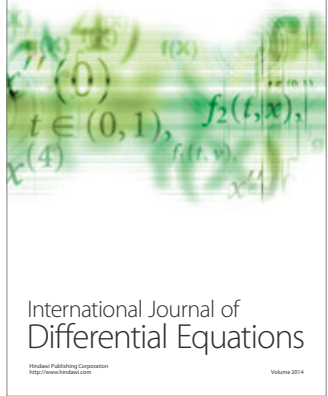
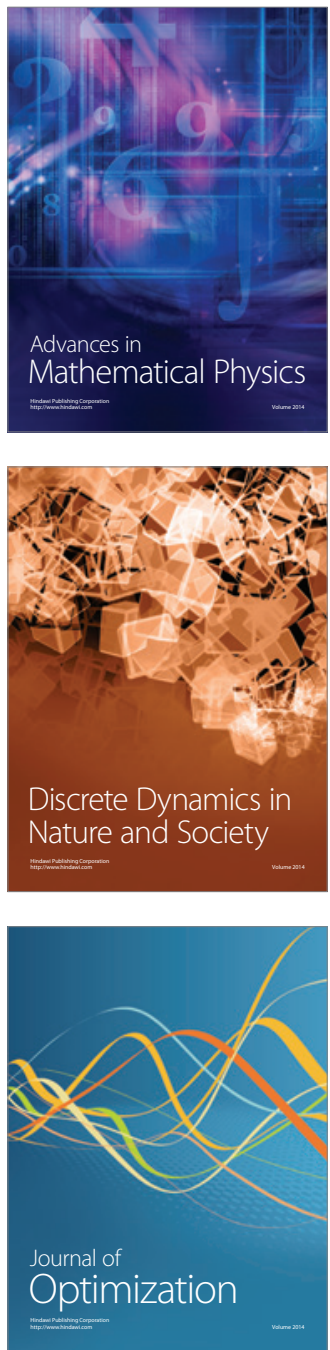